\title{
TÉCNICA QUIRÚRGICA DE GASTROYEYUNOSTOMÍA COMO VÍA ALTERNA PARA EL PASAJE DE ALIMENTOS EN PERROS CON ESTENOSIS PILÓRICA EXPERIMENTAL
}

\author{
Víctor Mamani P. ${ }^{1}$, Alfonso Chavera C. ${ }^{2}$ y Víctor Fernández A. ${ }^{3}$
}

\section{Abstract}

\begin{abstract}
A surgery technique of gastroyeyunostomy was applied in dogs with experimental pyloric stenosis to observe the transit of chyle through the new path, and to evaluate the adverse effects on the animal. Ten crossbred dogs ( 5 female and 5 male) of 2-3 years old were surgically pyloric stenosed and the gastroyeyunostomy was performed. Animals were clinically evaluated for 35 days, and then, contrast radiographs were taken before necropsy. The post-surgery clinical observations shown an adequate recovery by day 10 after surgery. Vomit, an important clinical sign was evaluated. Animals were grouped as "bad" for recurrent vomits, "good" for occasional vomits, and "excellent" for animals apparently normal. 33\% of dogs were considered as "good" and $67 \%$ as "excellent". An adequate outflow of contrast liquid through the anastomosed organs was observed in all animals. There were no lesions, infections or complications in the abdominal cavity at necropsy. All animals presented gastric biliar regurgitation without serious lesions in the gastric mucosa. Therefore, this technique could be considered simple, useful, with minimal possibilities of iatrogenic trauma.
\end{abstract}

Key words: gastroyeyunostomy, pyloric stenosis, recurrent vomits, canine, contrast radiograph

\section{Resumen}

Se expone la técnica quirúrgica de gastroyeyunostomía en caninos con estenosis pilórica experimental para observar el tránsito de quilo a través de esta vía alterna y evaluar los posibles efectos adversos que ocasione en el animal. Se utilizaron 10 caninos mestizos ( 5 hembras y 5 machos) de 2 a 3 años de edad, a los que se les estenosó el píloro utilizando una ligadura de seda y luego se les realizó la técnica quirúrgica de gastroyeyunostomía. Se evaluaron por 35 días post-operatorios, y al final se les tomó radiografías de contraste y se les practicó la necropsia. La observación clínica mostró que los animales se recuperaron dentro de los 10 días de la cirugía. El vómito, signo cardinal en los casos de estenosis pilórica fue evaluado, y los animales se agruparon por el análisis en base a este signo como "malos" si el vómito era recurrente, "buenos" si presentaban vómitos ocasionales y "excelentes" si estaban aparentemente normales. De esta forma, el 33\% de los perros fueron catalogados como "buenos" y el 67\% como "excelentes". En las tomas radiográficas seriadas de contraste se observó el pasaje del medio de contraste a través de la anastomosis en el total de los animales. En la necropsia no se observaron lesiones que indiquen infecciones o complicaciones post-operatorias serias en la cavidad y órganos abdominales. Todos los animales presentaron regurgitación biliar al estómago pero

\footnotetext{
${ }^{1}$ Facultad de Veterinaria y Zootecnia, Universidad Peruana Cayetano Heredia

${ }^{2}$ Laboratorio de Histo-Patología Animal,FMV-UNMSM.E-mail: achavera03@yahoo.com

${ }^{3}$ Clínica de Animales Menores, FMV-UNMSM
} 
a pesar de ello no hubo alteraciones aparentes en la mucosa gástrica. Para efectos del presente trabajo, se concluye que la técnica quirúrgica de gastroyeyunostomía es sencilla, útil y radical, en cuya realización se presentan pocas probabilidades de producir iatrogenia.

Palabras clave : gastroyeyunostomía, estenosis pilórica, vómitos recurrentes, caninos, radiografías de contraste

\section{INTRODUCCIÓN}

En los animales de compañía se presentan patologías pilóricas que obstruyen el pasaje de alimentos hacia el duodeno, teniendo como signo característico el vómito y por consiguiente el deterioro del estado de salud del animal, pudiendo llegar a ocasionar la muerte.

Una de las patologías más comunes es la llamada Gastropatía Hipertrófica Pilórica Crónica, frecuente en perros y en gatos, sobre todo en la raza Siamés. No tiene una etiología definida y puede deberse a causas genéticas, hormonales (hipergastrinemia) e infecciosas (Helicobacter sp.) Estas patologías han sido reconocidas en otros animales domésticos como el caballo y la cabra (Mc Hill y Bolton, 1984); así como en seres humanos donde el cuadro clínico se desencadena pocos meses después del nacimiento.

El diagnóstico clínico es práctico y se basa en la historia clínica y en radiografías de contraste en las cuales se mide el tiempo de vaciado gástrico que no debe ser mayor de 4 horas. Las técnicas de diagnóstico actuales incluyen el diagnóstico ecográfico y el uso de la endoscopía con la cual se puede obtener biopsias.

El tratamiento definitivo es básicamente quirúrgico y para esto existen varias técnicas que van desde las simples como la piloromiotomía y la piloroplastía, hasta las más radicales que incluyen la gastroduodenostomía, la pilorectomía, Billroth I, Billroth II y la operación en Y de Roux, en las cuales se forma un puente entre el estómago y el yeyuno. Las técnicas quirúrgicas radicales disminuyen las posibilidades de recurrencia de la estenosis debido al aumento de la hipertrofia, como se han manifestado en la pilomiotomía y en la piloroplastía, y son de mejor aplicación en hipertrofias pilóricas muy extensas como las polipoides. Walter et al. (1985a) realizaron gastroyeyunostomías y pilorectomías en caninos con resultados satisfactorios, y Weaver et al. (1987) practicaron gastroyeyunostomías en humanos con cáncer pancreático que provocaban estenosis pilórica y duodenal, mejorando la calidad de vida de los pacientes.

Este estudio experimental en el área de Medicina Veterinaria se basa en la aplicación de una técnica quirúrgica radical como la gastroyeyunostomía en perros, que es utilizada en cirugía humana para solucionar problemas de retención de alimentos en el estómago ocasionados por múltiples patologías que estenosan el lúmen pilórico (como neoplasias gástricas y del páncreas y contracción de las úlceras cicatrizales). Esta cirugía mejora la calidad de vida de los pacientes humanos aunque se presentan problemas clínicos como el "dumping", úlceras gastroyeyunales, problemas nutricionales de absorción y hasta cambios electrofisiológicos gastroentéricos que traen consigo cambios motores.

El objetivo de este trabajo fue evaluar la gastroyeyunoanastomosis con el fin de solucionar problemas de retención del quilo ocasionado por estenosis pilórica, tomando como modelo a perros con estenosis pilórica experimental. 


\section{Materiales y Métodos}

\section{Animales}

El trabajo se llevó a cabo en el Hospital de Animales Menores de la Facultad de Medicina Veterinaria de la Universidad Nacional Mayor de San Marcos. Se utilizaron 5 perras y 5 perros con edades de 2 a 3 años, de raza mestiza, clínicamente normales y con un peso promedio de $15.4 \mathrm{~kg}$.

A los animales se les mantuvo en observación y se les realizaron las pruebas de riesgo quirúrgico preoperatorios por 7 días. Se mantuvieron en 24 horas de ayuno previo a la cirugía. El día de la cirugía se procedió a anestesiar al animal y a sujetarlo en decúbito dorsal, se afeitó y se procedió a la desinfección química con yodo povidona en espuma para el lavado y en solución para el enjuague.

\section{La intervención quirúrgica}

Se utilizó como preanestésico y relajador muscular la acepromacina en dosis de $0.04 \mathrm{mg} / \mathrm{kg}$ i.v y $15 \mathrm{~min}$ después, como anestésico disociativo intravenoso, a la ketamina $(100 \mathrm{mg} / \mathrm{ml})$ en dosis de $10 \mathrm{mg} / \mathrm{kg}$ de peso. Se inyectó atropina $(0.04 \mathrm{mg} / \mathrm{kg}$ i.m. para evitar la salivación excesiva. Durante la cirugía y hasta la recuperación de la anestesia se mantuvo al animal bajo fluidoterapia $(\mathrm{NaCl}$ al $0.9 \%)$. Se colocaron campos estériles a cada lado de la línea media ventral tomando como referencia el apéndice xifoides del esternón y la cicatriz umbilical, y una sábana abierta con abertura longitudinal en el centro que cubría toda la mesa de cirugía. pasos:

La cirugía comprendió los siguientes

? Se realizó una incisión longitudinal en la piel del abdomen ventral de aprox. $5 \mathrm{~cm}$ desde el apéndice xifoides hacia caudal.

? Se profundizó en los planos anatómicos de la piel, panículo adiposo y tejido subcutáneo.
? Se hizo una incisión de $5 \mathrm{~cm}$ en la línea alba con la tijera de Mayo guiada con los dedos. Las tijeras se emplearon en posición semicerrada y haciendo movimientos de progresión a lo largo de la línea alba. En algunas ocasiones se utilizó el separador de Balfour para exponer el abdomen.

? Se ubicó el estómago, se hizo tracción con la pinza de Babcock hasta encontrar la región pilórica, la cual se expuso fuera de la cavidad abdominal. Los vasos sanguíneos adyacentes a la pared serosa del lado mesentérico y antimesentérico se divulsionaron con la pinza de Kelly y se pasó el hilo de seda por debajo de ellos con la ayuda de esta pinza, haciendo una doble ligadura para estenosar la luz pilórica (Fig. 1). Se debió manipular delicadamente para no dañar el páncreas, aún cuando la estrechez pilórica se encuentra relativamente alejada del páncreas y conductos biliares.

? Se expuso el yeyuno, que se encuentra encima del colon transverso a la altura de la $2^{a}$ vértebra lumbar, a una distancia aprox. de $15 \mathrm{~cm}$ del ligamento de Treitz. Esta región del yeyuno se colocó paralelamente a la región antral del estómago y se suturaron $6 \mathrm{~cm}$ con puntos continuos simples seromusculares a lo largo de los lados mesentéricos de ambos, utilizándose hilo de poliéster. En ambos extremos se dejaron cabos largos.

? El yeyuno y el estómago se incidieron con el bisturí, lo suficiente para que ingrese la punta de la tijera Metzenbaum. Se cortó una distancia aprox. de $5 \mathrm{~mm}$ de la línea de sutura seromuscular y se iniciaron los puntos continuos entrelazados desde el centro del corte hacia los extremos con dos hilos reabsorbibles de poliglactina.

? Luego de hacer los nudos en cada extremo, se continuó la sutura con puntos de Connell, desde los extremos al centro, a 


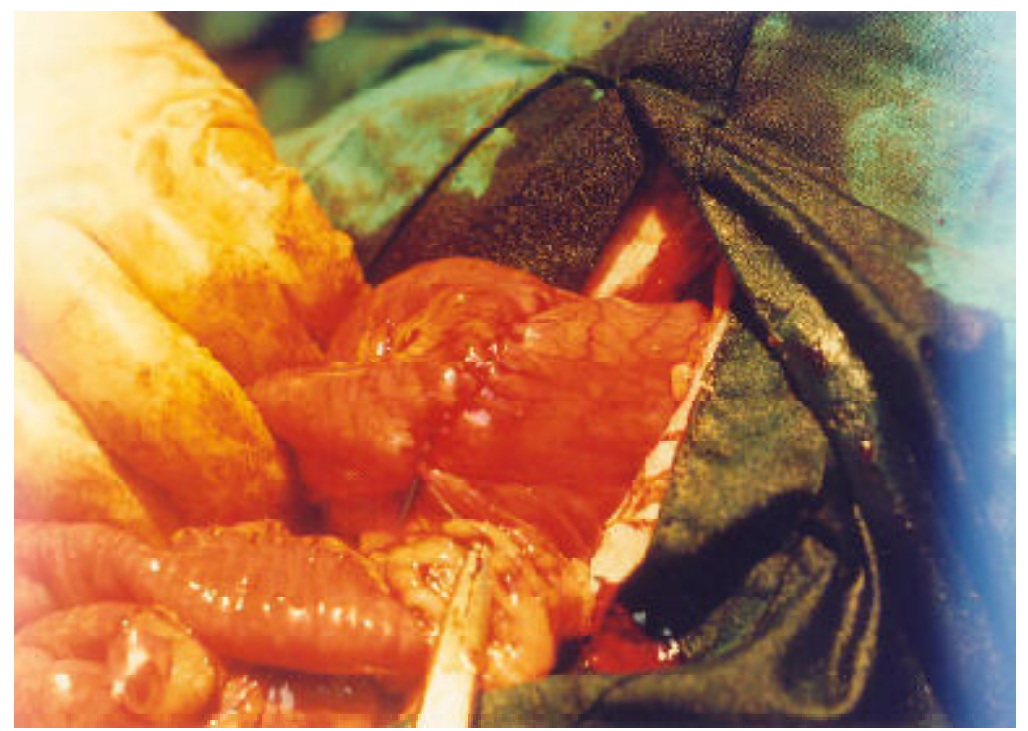

Figura 1. Gastroyeyunoanastomosis finalizada

fin de que al anudarse distorsionen menos el tejido.

? La capa seromuscular se suturó con puntos continuos utilizándose poliéster (Ethibond ${ }^{\circledR} 3-0$ ). Se introdujeron delicadamente las regiones de los órganos expuestos y se procedió con la sutura de la laparotomía.

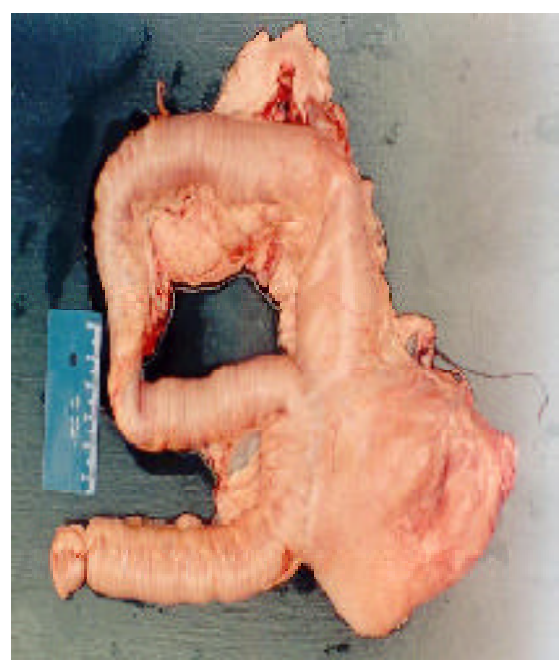

Figura 2. Vista panorámica postnecropsia de la gastroyeyunostomía

\section{Control clínico post-operatorio}

Los pacientes se mantuvieron con fluidoterapia a base de $\mathrm{NaCl}$ al $0.9 \%$ hasta que se observaron los signos clínicos de salida gradual de los planos anestésicos. El cuidado post-operatorio incluyó ayuno de 24 a 48 horas, fluidoterapia ( $\mathrm{NaCl}$ al $0.9 \%, 40 \mathrm{ml} /$ kg i.v., dos veces al día), administración de antibióticos (penicilina asociada a estreptomicina intramuscular por 5 días) y ranitidina ( $0.5 \mathrm{mg} / \mathrm{kg}$ i.v., dos veces al día por 3 días).

Se llevó el control clínico postoperatorio durante 35 días anotándose el estado general del animal, temperatura corporal, frecuencia respiratoria y cardiaca, defecación, apetito y emesis. Los puntos de sutura se retiraron a los 10 días de la operación.

El vómito, signo principal en los casos de estenosis pilórica, se evaluó al final del estudio considerándose tres grupos: "malo" si presentaron signos clínicos severos de estenosis pilórica según Walter et al. (1985b), "bueno" si presentaron signos ocasionales de enfermedad gástrica, como los vómitos, y "excelente" si los perros se encontraban aparentemente normales.

\section{Tomas radiográficas de contraste}

Se realizaron tomas radiográficas laterolaterales y ventrodorsales a los 35 días post-cirugía, utilizándose como medio de 
contraste el sulfato de bario en dosis de 100 $\mathrm{ml} /$ animal. Las tomas radiográficas se realizaron a los $0,30,60$ y 120 minutos de la administración del líquido de contraste.

\section{Necropsias}

Los perros fueron sacrificados a los 35 días de la cirugía con pentobarbital sódico $(100 \mathrm{mg} / \mathrm{kg})$ y se les practicó la necropsia, realizándose las siguientes observaciones: estado general, focos infecciosos, peritonitis, adherencias, estenosis y continuidad de la luz de la anastomosis.

\section{Resultados}

Todos los animales se encontraron aparentemente normales, defecaron y tuvieron apetito en los días subsiguientes a la cirugía. No se evidenciaron alteraciones que indiquen procesos inflamatorios o infecciosos durante los 35 días postquirúrgicos.

Tres de los 10 perros fueron catalogados como "buenos", siete como "excelentes" y ninguno como "malo" con relación a la ocurrencia del vómito, indicando que los animales no presentaron retención del quilo ocasionado por la piloroestenosis experimental.

Los hallazgos radiográficos de contraste de todos los animales mostraron el transito de sulfato de bario del estómago a los intestinos, comprobándose de esta manera la viabilidad de la anastomosis gastroyeyunal.

En la necropsia no se observaron lesiones indicativas de infecciones o complicaciones post-operatorias serias en la cavidad abdominal o en órganos que se encuentran ubicados en ella (Fig. 2). Se pudo observar que se obtuvo una anastomosis de tamaño adecuado que permitió el paso de los alimentos. Todos los animales tuvieron regurgitación biliar al estómago, la que, al parecer, no ocasionó complicaciones clínicas. No se evidenciaron daños en la mucosa estomacal, en la línea anastomótica, ni en la mucosa yeyunal después de los 35 días postquirúrgicos.

\section{Discusión}

Según trabajos realizados por Gualtieri et al. (1994), Mattiensen y Walter (1986) y Walter et al. (1985b), las técnicas más radicales han tenido mejores resultados a largo plazo que otras, como la piloroplastía o piloromiotomía que son más sencillas. La gastroyeyunostomía, técnica radical utilizada en este estudio, tuvo resultados satisfactorios ya que la presentación de vómito, que es el signo clínico cardinal, fue mínima hasta el final del periodo de evaluación y no se encontraron casos de úlceras yeyunales que si fueron reportados en los trabajos realizados por Marckowitz et al. (1969).

En el presente trabajo no se utilizaron fármacos como sucralfato o sales de bismuto como protectores de la mucosa gastrointestinal, recomendados por diversos autores (Ettinger, 1992; Gualtieri et al., 1994; Goodman y Hillman, 1994; Strombeck, 1993), ya que se deseaba observar el efecto directo de los ácidos gástricos sobre la mucosa yeyunal.

En las técnicas de gastroduodenostomía, pilorectomía, gastroyeyunostomía Billroth I y Billroth II se hace un manipuleo muy cercano al páncreas, conductos biliares $\mathrm{y}$ vasos mayores, que pueden causar problemas iatrogénicos (Walter et al., 1985b). En este estudio, cuando se realizó la gastroyeyunostomía, se expuso la zona avascular del estómago así como la primera asa yeyunal, las cuales no tienen peligro de dañar órganos importantes del sistema gastrointestinal, además que se pueden tener expuestos por mayor tiempo durante la cirugía, siendo por lo tanto una técnica de fácil ejecución y que disminuye el riesgo de causar problemas iatrogénicos que complicarían la cirugía.

Revisiones de literatura en medicina y cirugía humana exponen ciertos problemas 
en los pacientes a los que se ha realizado la técnica de gastroyeyunostomía. Estos incluyen pancreatitis, gastritis por reflujo alcalino y de ácidos biliares al estómago, el síndrome de "dumping" y problemas nutricionales. El reflujo de bilis y jugo pancreático causa gastritis con severo dolor en el epigastrio. Estos problemas no se observaron en los perros del presente trabajo, corroborando lo expuesto por Mattiensen y Walter (1986) y Walter et al. (1985b) sobre la resistencia natural de los perros a los problemas mencionados (Greger y Windhorst, 1997). También se han detectado problemas en la absorción de microelementos y oligoelementos como el calcio y el cobre en pacientes humanos operados que tienen un largo periodo post-quirúrgico, pero que no fueron evaluados en este estudio (Walter et al., 1985b).

Todos los perros tuvieron problemas de regurgitación del intestino al estómago con contenido biliar y pancreático. A pesar de esto, no se presentaron problemas clínicos serios (Schwartz, 1996). Otros trabajos manifiestan que para disminuir los riesgos a largo plazo se debe administrar protectores de la mucosa tales como sucralfato o sales de bismuto por largos periodos. Aquí no se presentaron cambios en la mucosa en los 35 días del estudio a pesar de no haber utilizado protectores de mucosa, lo que no significa que en un tiempo más prolongado pudiera llegar a ocurrir (Ettinger, 1992).

Una forma práctica de observar la viabilidad in vivo de la anastomosis fueron las radiografías de contraste, las cuales permitieron comprobar el vaciado del contenido gástrico al yeyuno. En los trabajos revisados, estas radiografías sólo fueron tomadas para el diagnóstico de estenosis pilórica (Bellenger et al., 1990; Gualtieri et al., 1994; Mattiensen y Walter, 1986; Walter et al., 1985a), en tanto que en este estudio se tuvo que realizar para comprobar la viabilidad de la gastroyeyunoanastomosis en los perros.

En la necropsia no se observaron cambios aparentes en las mucosas gastro- entéricas y el tamaño de la luz anastomótica fue el adecuado. Sin embargo, es importante recalcar que el hilo reabsorvible utilizado ya no se encontraba en el día 35, por lo que se considera importante que la segunda línea de sutura se haga con material no reabsorvible, el cual se encontró íntegro y sujetando la anastomosis. Esto sería recomendable ya que los perros con píloroestenosis o aquellos a los que se les ha practicado cirugías gástricas suelen presentar vómito que pueden romper suturas debilitadas o en vías de reabsorción (Schwartz,1996).

\section{Conclusiones}

- La ejecución de la técnica quirúrgica es sencilla, iatrogénica y tiene pocas posibilidades de producir recurrencia de la estenosis y de los signos clínicos característicos de estenosis pilórica.

- La ausencia de signos clínicos adversos, el buen estado general post-quirúrgico, la observación de la viabilidad in vivo y post mortem de la anastomosis y la ausencia de hallazgos patológicos en los órganos gastrointestinales hacen que esta técnica quirúrgica sea útil para mejorar la calidad de vida de los animales con problemas de estenosis pilórica.

\section{Literatura Citada}

1. Bellenger, C; J. Maddison; G. McPherson; J. Ilkiw. 1990. Chronic hypertrophic pyloric gastropathy in 14 dogs. Aust. Vet. J. 67: 317-320.

2. Ettinger, S. 1992. Tratado de Medicina Interna Veterinaria. $3^{a}$ ed. p 1449-1458. Ed. Intermédica. Argentina.

4. Goodman, W.; R. Hillman. 1994. Bases Farmacológicas de la Terapéutica. $8^{\mathrm{a}}$ ed. $\mathrm{p}$ 965-971. Ed. Interamericana. México.

5. Greger, R.; U. Windhorst. 1997. Comprehensive Human Physiology. $3^{\mathrm{a}}$ ed. p 1245-1249. Ed. Springer. Alemania. 
6. Gualtieri, M; M. Monzeglio; E. Scanziani; C. Domeneghini. 1994. Pyloric hyperplastic polyps in the French Bulldog. Europ. J. Comp. Anim. Prac. 8: 33-42.

7. Marckowit, J; J. Archibald; G. Downie. 1967. Cirugía experimental. $5^{a}$ ed. p 146147. Ed. Interamericana. México.

8. Mc Hill, C.; J. Bolton. 1984. Gastric retention associated with a pyloric mass in two horses. Aust. Vet. J. 61: 190-191.

9. Matthiensen, D.; M. Walter. 1986. Surgycal treatment of chronic hypertrophic pyloric gastropathy in 45 dogs. JAAHA 22: 241-247.

10. Schwartz, E. 1996. Texto de cirugía. $5^{\mathrm{a}}$ ed. p 975-977. Ed. Interamericana. México.
11. Strombeck, D. 1993. Gastroenterología en pequeños animales. $3^{3}$ ed. p 94-97. Ed. Intermédica. Argentina.

12. Walter, M; M. Goldschmidt; M. Stone; J. Dougherty; D. Matthiensen. 1985a. Chronic hypertrophic pyloric gastropathy as a cause of pyloric obstruction in the dog. JAVMA 186: 157-161.

13. Walter, M; D. Matthiensen; J. Stone. 1985b. Pylorectomy and gastroduodenostomy in the dog: Technique and clinical results in 28 cases. JAVMA 187: 909-915.

14. Weaver, D; R.Wiencek; D. Bowman; A. Walt. 1987. Gastrojejunostomy: Is helpful for patients with pancreatic cancer? Surgery 102: 808-813. 